\title{
Visual and olfactory oddity learning in rats: What evidence is necessary to show conceptual behavior?
}

\author{
ROGER K. THOMAS and LINDA M. NOBLE \\ University of Georgia, Athens, Georgia
}

\begin{abstract}
Experiment 1 involved the use of plastic and wooden objects and trial-unique problems. The rats performed successfully on nonconceptual oddity problems given before and after conceptual training, showing that the testing conditions were suitable, but they showed chance performances on the trial-unique problems. Experiment 2 involved the use of olfactory discriminanda. Five pretraining problems and 300 unique five-trial problems were presented. Two of 3 rats performed better than chance on Trial 2 and on Trials 3-5, but all performed at chance levels on Trial 1 throughout. The data suggest that the rats responded to specific odors on Trials $2-5$ following the Trial 1 experience, as opposed to responding conceptually to the "odd" odor. Had they responded conceptually to odd odors, they should have performed better than chance on Trial 1 . These findings and the general logical argument that they support are considered in the context of the numerous inconclusive reports of the use of the oddity concept by nonprimate animals.
\end{abstract}

In the context of the evolution of intelligence, the oddity concept is an important example of relative class concepts in the hierarchy of intellectual abilities (Thomas, 1980). Tasks involving the oddity concept have been widely used to study the comparative learning abilities of animals. Oddity tasks can be adapted to each species, and can therefore be relatively free of confounding contextual variables (species differences in sensory and motor capacities, motivational variables, etc.). There have been numerous reports that rats (Wodinsky \& Bitterman, 1953), cats (Warren, 1960), canaries (Pastore, 1954), and pigeons (Zentall \& Hogan, 1974), to mention only a few examples, have used the oddity concept.

However, these and other such studies have been repeatedly criticized on the grounds that their experimental procedures allowed nonconceptual performances (e.g., see critiques by French, 1965; Strong \& Hedges, 1966; Thomas \& Boyd, 1973; Thomas \& Frost, 1983). Furthermore, most of the questionable reports postdate D. R. Meyer and Harlow's (1949) careful consideration of the kind of evidence that is necessary to demonstrate an animal's use of the oddity concept.

In view of the importance of the oddity concept and the continuing publication of questionable claims of its use by animals (e.g., Pisacreta, Lefave, Lesneski, \& Potter, 1985), another presentation of the evidence necessary to demonstrate an animal's use of the oddity concept is in order. The present study provides empirical evidence on

Address requests for reprints to Roger $\mathbf{K}$. Thomas, Department of Psychology, University of Georgia, Athens, GA 30602 . Linda M. Noble's present address is Department of Psychology, Kennesaw College, Marietta, GA 30060 . the most crucial point in the argument-namely, the need for first-trial transfer data. Although the present argument and the supporting evidence are presented primarily in terms of the oddity concept, the argument is applicable to all tests of conceptual behavior.

As evidence for the conceptual use of oddity, investigators have typically reported facilitated transfer of training with new oddity problems. However, they usually have not reported whether the facilitated transfer occurred on the first trials on which new oddity problems were presented. Claims for conceptual behavior based on transfer data that do not include separate analyses of first-trial performances are inconclusive. The animal might learn, even in one trial, to associate reinforcement with specific properties (color, shape, or size) of the object in which the oddity concept is manifested, as opposed to associating reinforcement with the relative property of "oddity." Evidence of facilitated transfer in the absence of first-trial data may reflect acquisition of the learning-set process (Harlow, 1949). Whether the learning-set process is conceptual is not an issue here, but the use of the oddity concept and the use of the learning-set process are not equivalent.

An exception to the argument that first-trial data are necessary can be found in the report by Lombardi, Fachinelli, and Delius (1984). Their conclusion that pigeons used the oddity concept was based on a kind of evidence that they were apparently the first to report. They reported better-than-chance performances on a test set of oddity problems that were never associated with reinforcement. The test problems were presented together with a training set of oddity problems; responses to the odd stimulus in the training set were reinforced. Since there was no opportunity to associate specific properties of the 
stimuli in the test set with reinforcement, the better-thanchance performances on the test set were presumably the result of transfer of the oddity concept from the training set. The study by Lombardi et al. provides the best evidence to date that a nonprimate animal can respond to oddity conceptually.

Two experimental studies using rats are reported here. The first experiment used visual exemplars of the oddity concept, and the second experiment used olfactory exemplars of the oddity concept.

\section{EXPERIMENT 1: VISUAL ODDITY}

Previous studies of visual oddity learning in rats used what Levinson (cited in French, 1965) called the "oneodd" (Koronakos \& Arnold, 1957) or "two-odd" " procedures (Wodinsky \& Bitterman, 1953). These procedures confound oddity as a cue with nonconceptual cues. The one-odd procedure uses the same three discriminanda (symbolically: $\mathrm{ABB}$ ) until the animal meets criterion or fails to learn the problem. At least two nonconceptual solutions are possible: (1) The animal might learn merely to distinguish specific physical properties of $A$ from those of $B$ and associate those properties of $A$ with reinforcement, as opposed to learning that $A$ is the stimulus in which the relational property of oddity is manifested, or (2) it might learn to associate reinforcement with the A component of the three specific patterns that result from the typical side-by-side presentations of the discriminanda (i.e, $\mathrm{ABB}, \mathrm{BAB}$, and $\mathrm{BBA}$ ).

In an attempt to avoid the nonconceptual solutions afforded by the one-odd procedure, Robinson (1933) introduced the two-odd procedure (Levinson's term). The two-odd problem randomly offers A or B as the odd stimulus, and thereby eliminates the first solution described above for the one-odd procedure. However, it does not eliminate the specific-pattern solution, although the number of specific patterns to be learned is doubled (i.e., ABB, $\mathrm{BAB}, \mathrm{BBA}, \mathrm{BAA}, \mathrm{ABA}$, and AAB). Although it is possible that the animal learns the oddity concept, such a conclusion could not be reached, because the nonconceptual, specific-pattern solution remains a possibility.

The previous studies of visual oddity learning in the rat, as noted above, used the one-odd or two-odd procedures. In the present study, after the rats had been pretrained on 10 one-odd problems, a new oddity problem was used on each trial for a total of 300 problems and trials. The previous studies used two-dimensional stimuli. Miscellaneous plastic and wooden objects were used in the present experiment on the basis of research with nonhuman primates that suggested that performances were better with objects as discriminanda than with twodimensional stimuli (D. R. Meyer, Treichler, \& P. M. Meyer, 1965).

\section{Method}

Subjects. Four female hooded rats, derived from the Long-Evans strain (Rattus norvegicus) and bred in the University of Georgia's rat colony, were used. The rats were 90-120 days old at the start of the experiment. They were housed in individual cages and maintained on a light:dark cycle, with darkness occurring between 8:00 a.m. and 8:00 p.m. local time. Testing took place during the dark phase. The rats were deprived of food for $48 \mathrm{~h}$ prior to the beginning of the study, but were thereafter fed a daily ration of 12-15 g of Purina Laboratory Rodent Chow. Their access to water was unrestricted.

Apparatus and Stimuli. The testing apparatus had two compartments: a holding chamber (HC) for the rat and a stimulusreinforcement chamber (SRC). The sides and the top of the HC were constructed of wood and painted black. The floor of the HC was constructed of stainless steel rods spaced $1.25 \mathrm{~cm}$ apart across the width of the chamber. The inside dimensions of the $\mathrm{HC}$ were $31 \mathrm{~cm}$ (length) $\times 29 \mathrm{~cm}$ (width) $\times 20 \mathrm{~cm}$ (height). The wall facing the SRC had an aperture across its width that was $7 \mathrm{~cm}$ from the grid floor. This aperture could be closed by a guillotine door.

The SRC was constructed of wood and painted medium gray. Its inside dimensions were $29 \mathrm{~cm}$ (width) $\times 20 \mathrm{~cm}$ (height) $\times$ $13.5 \mathrm{~cm}$ (length). The SRC was designed to be juxtaposed to the HC. It had no wall on the side closest to the HC; instead, it shared the HC's wall, which had the aperture described above. The SRC's wall opposite the side with the aperture had a guillotine door that could be opened to permit the experimenter to set up the stimuli on the stimulus tray. The stimulus tray could be reached by the rat through the aperture in the $\mathrm{HC}$.

The stimulus tray, which was constructed of wood and painted medium gray, had three food wells with diameters of $2.5 \mathrm{~cm}$ and depths of $0.5 \mathrm{~cm}$; the centers of the outer food wells were $4 \mathrm{~cm}$ from the sides of the tray and the center-to-center distance between the food wells was $8 \mathrm{~cm}$. Each food well was covered by a small medium-gray board $(3.5 \mathrm{~cm} \times 22.5 \mathrm{~cm} \times 0.5 \mathrm{~cm})$ that could be moved back and forth to cover or uncover the food well. Although there were three food wells, only the outer two were used in the present study.

The nonodd stimuli were chosen from an available pool of approximately $\mathbf{5 0 0}$ distinct pairs of identical objects. The odd stimuli were chosen either as one of the objects from an identical pair or as single objects from a pool of several hundred objects. The stimuli on a given trial were selected from these pools of objects, with the restriction that the odd stimulus differ from the nonodd stimuli in color, shape, and size. The room in which the animals were tested was illuminated only by the daylight that entered through a closed venetian blind in a single window. The stimuli on the testing tray were illuminated by a fluorescent desk lamp located just above and behind the holding chamber.

Pretraining procedures. (1) For the first 3 days, the rat was placed in the HC with the guillotine door between it and the SRC in the open position. Two food pellets were placed in a randomly selected outer food well and the rat was allowed to remain in the HC until it consumed both pellets. (2) For the next 3 days, the animal was placed in the $\mathrm{HC}$ with the guillotine door closed. The door was raised after $60 \mathrm{sec}$ allowing access to the food; the animal was allowed to remain until it consumed the food. (3) The first pretraining oddity problem was introduced on the 7th testing day. Hereafter, in both pretraining and training, 20 trials per day were administered. Initially, the food wells were not covered by the small boards, and the objects were placed directly behind the food wells. On Day 2 of the first pretraining problem, the objects covered about one-third of the food well; on Day 3, about one-half; and on Day 4, about two-thirds. Beginning on Day 5, the objects completely covered the food wells, and the animal continued to receive training on the first problem until it reached a criterion of 18 correct in a 20-trial session. The position of the odd object-the correct choicewas limited to the left or right food well and was determined by the Fellows (1967) series. The rat had to displace the odd object before the experimenter would slide back the board that covered 
the food well and the reinforcers. Both outer food wells were baited as a control for possible odor cues from the food pellets. (4) The rat was given a total of 10 pretraining problems, and training on each problem continued until the rat met a criterion of 18 correct in a 20-trial session. A correction procedure was used in both pretraining and training in which, in the event of an error, the trial was readministered until a correct response was seen or until a total of five administrations of the trial had been given. For purposes of data analysis, only the response to the initial presentation of a trial was used.

Conceptual oddity training. Following the attainment of criterion on the 10th pretraining problem, training was continued by giving the animal a newly generated oddity problem on each trial. Twenty problems were given per day. We had intended to train the rats to the criterion of 18 correct in 20 trials, but when chance performance was evident in all rats at the end of 300 problems, training was terminated.

Postconceptual oddity training test. Because the rats were at chance levels after 300 trials of conceptual oddity training, it was decided post hoc to administer one more one-odd problem as a test of possible motivational decline, attentional deficits, and so forth.

\section{Results}

Pretraining. All rats reached criterion on all 10 pretraining problems. The number of trials to criterion for each rat on each problem is shown in Table 1. There was no evidence of interproblem improvement, although Problem 6 appeared to be the easiest and Problem 3 the most difficult. Neither was there evidence of much difference among the rats. The total number of trials to criterion on the 10 problems for the 4 rats were 1,040 (Rat L1), 1,040 (L10), 940 (L11), and 1,070 (L12).

Conceptual oddity training. All rats were performing at chance levels $(50 \%)$ when the experiment was terminated after 300 problems had been administered. Table 2 shows the number of errors for each rat in each 20-trial session. According to the binomial approximation test (M. E. Meyer, 1976), the probability that four errors would occur by chance in 20 trials is .007 , and that five errors would occur by chance is .022 . Rat L12 had four errors in Session 3, Rat L10 had five errors in Session 10, and Rat L11 had five errors in Sessions 7 and 9.

Postconceptual oddity training test. Rats L1 and L10 reached criterion in 120 trials. Rat L11 did not reach criterion in the $\mathbf{2 0 0}$ trials allowed, although it performed as

Table 1

Trials to Criterion by Each Rat, and Median Trials to Criterion on the 10 Pretraining Problems

\begin{tabular}{crrrrr}
\hline & \multicolumn{4}{c}{ Rat } & \\
\cline { 2 - 5 } Problem & L1 & L10 & L11 & L12 & Median \\
\hline 1 & 140 & 60 & 120 & 80 & 100 \\
2 & 80 & 120 & 100 & 140 & 110 \\
3 & 180 & 180 & 180 & 180 & 180 \\
4 & 80 & 80 & 20 & 80 & 80 \\
5 & 100 & 120 & 120 & 80 & 110 \\
6 & 40 & 40 & 60 & 60 & 50 \\
7 & 160 & 120 & 80 & 60 & 100 \\
8 & 120 & 140 & 40 & 200 & 130 \\
9 & 60 & 100 & 160 & 160 & 130 \\
10 & 80 & 80 & 60 & 120 & 80 \\
\hline
\end{tabular}

Table 2

Errors Per 20-Trial Session for Each Rat, and Median Errors Per Session During Conceptual Oddity Training

\begin{tabular}{crrrrc}
\hline & \multicolumn{5}{c}{ Rat } \\
\cline { 2 - 5 } Session & L1 & L10 & L11 & L12 & Median \\
\hline 1 & 11 & 11 & 12 & 10 & 11 \\
2 & 11 & 10 & 12 & 11 & 11 \\
3 & 13 & 12 & 15 & 4 & 12.5 \\
4 & 11 & 6 & 6 & 6 & 6 \\
5 & 9 & 13 & 8 & 7 & 8.5 \\
6 & 9 & 12 & 8 & 9 & 9 \\
7 & 10 & 10 & 5 & 8 & 9 \\
8 & 12 & 12 & 10 & 8 & 11 \\
9 & 12 & 7 & 5 & 11 & 9 \\
10 & 6 & 5 & 12 & 6 & 6 \\
11 & 7 & 10 & 10 & 11 & 10 \\
12 & 9 & 14 & 11 & 8 & 10 \\
13 & 10 & 15 & 12 & 10 & 11 \\
14 & 9 & 15 & 9 & 11 & 10 \\
15 & 7 & 9 & 11 & 8 & 8.5 \\
\hline
\end{tabular}

well as $80 \%$ correct (during the 9th session). Rat L12 also did not reach criterion in the 200 trials allowed, although it had $85 \%$ correct in the 9 th session and $80 \%$ in the 10 th session.

\section{Discussion}

The data from the one-odd pretraining problems and the one-odd problem used as a posttest show that the rats were capable of performing successfully in our testing situation when the tasks were within their capacities. Furthermore, despite the chance performances seen at the end of conceptual oddity training, there were four sessions involving 3 rats in which the performances may have been better than chance. Of course, these four sessions occurred among 60 such sessions, which increases the likelihood that these four were due to chance. In any event, it is possible that different training procedures might have resulted in a more successful demonstration of the rat's conceptual use of oddity, but on the basis of the results of the present experiment, it seems best to conclude that this one was not successful.

\section{EXPERIMENT 2: OLFACTORY ODDITY}

Langworthy and Jennings (1972), who studied olfactory oddity learning in rats, introduced an easy way to present discriminanda in the olfactory modality. Their discriminanda were ping-pong balls saturated with odors obtained from commercially available food flavorings. Three balls were used on each trial, two with the same odor. The appropriate response was to nudge aside the ball with the odd odor to obtain a food reinforcer. Langworthy and Jennings gave the rats a total of 30 oddity problems, each to a criterion of 16 correct in 20 trials or until 100 trials had been given. In addition to reporting considerable interproblem improvement, they reported first-trial performances. Although the heading for the table that shows the first-trial results is confusing, a reasonable interpretion is that correct responses were seen on $38(69 \%)$ of 
the 55 first trials. This figure can be obtained by multiplying the number of rats (11) by the number of first trials of the last five problems. Langworthy and Jennings did not report whether the response rate of $69 \%$ correct differed from chance $(50 \%)$, but our application of the binomial approximation (following M. E. Meyer, 1976) suggested that it did $(p<.004)$.

In addition to the table, another point of concern is the possible lack of control for odor cues emanating from the food reinforcers. Apparently, Langworthy and Jennings (1972) baited only the correct food well on each trial. Control rats that responded to unscented ping-pong balls performed at chance levels, suggesting that the odor of the food was not a cue. However, it is possible that attention to the odors from the food reinforcers was enhanced for the experimental rats as a function of their having learned to respond to odor discriminanda. Specifically, it is suggested that the rats might have smelled the food reinforcer and used that as the cue to displace the odd ball; that is, they may have responded directly to the food without having attended to the discriminanda.

There were three major differences between their experiment and ours. First, they administered 30 problems, each to a criterion of 16 correct responses in 20 trials or a maximum of 100 trials, whereas we gave 300 five-trial problems. Second, they constructed their problems from a stimulus pool of 8 odors, whereas we used 16. Third, they apparently baited only the food well associated with the correct choice, whereas we baited both food wells.

\section{Method}

Subjects. Three male hooded rats were used. Otherwise, the subjects' conditions were the same as those in Experiment 1.

Apparatus and Stimuli. The apparatus was the same as that used in Experiment 1 with two modifications: (1) The stimulus tray contained a front wall that mated with the aperture described in Experiment 1 . This wall had three portals, each $4 \times 5 \mathrm{~cm}$, allowing access to the food wells. The portals were intended to minimize forward rolling of the ping-pong balls. The rat could nudge a ball through the portal, causing it to roll back. (2) The small boards used to cover the food wells now contained a small indentation centered over the food well to hold the ball in place until it had been nudged.

The discriminanda were odor-exposed ping-pong balls. To prepare the ping-pong balls with the odors, one-quart Mason brand food-storage jars, which could be closed tightly against a rubber gasket, were used to hold the balls and the odoriferous substances. Seven drops of an odoriferous substance were placed in the bottom of a clean jar. A screen wire was installed to prevent direct contact of the substance with the balls, and two balls were placed in each jar. Throughout the study, the balls were kept in the same jar, and the odoriferous substance was replenished as needed. Sixteen substances were used. Fifteen were the following McCormick brand food flavorings: almond, anise, banana, black walnut, butter, chocolate, coconut, lemon, maple, mint/peppermint, orange, pineapple, rum, strawberry, and vanilla. The 16th substance was Eckerd brand oil of wintergreen. Sixteen substances, used 2 at a time when either substance may be the odd stimulus, permit the construction of 240 odor-unique problems. When the positions of the odd and nonodd stimuli are taken into account, 960 unique configurations are possible.
Pretraining. Procedures comparable to those in Experiment 1 were used, except that 5 instead of 10 pretraining problems were administered. Each problem was administered at a rate of 20 trials per day until the animal responded correctly on $80 \%$ or more of the trials for two successive sessions, or until it responded correctly on $90 \%$ of the trials in a single session.

Oddity learning-set training. Beginning with the session following the attainment of criterion on the fifth pretraining problem, the animals were given four new problems each session until a total of 300 new problems had been given. Each problem was administered for five trials.

\section{Results and Discussion}

Pretraining. The number of trials to criterion for each rat and the median trials to criterion for the five pretraining problems are shown in Table 3 . The $90 \%$ correct criterion was met in all cases except that of Rat 1 on the first problem; Rat 1 met the criterion of 2 days at $80 \%$ correct or better by having $85 \%$ correct on Day 4 and $80 \%$ correct on Day 5 . There was general improvement in performance across problems as reflected in the median trials to criterion. The results seen on the five pretraining problems here may be compared directly to Langworthy and Jennings's (1972) results on their first five problems; the interproblem improvement seen here is very similar to what they reported. Another similarity between their study and ours is the finding that the rats did not consistently respond correctly on the first trials of the first five problems.

Oddity learning-set training. It was generally found that the rats performed at chance levels on Trial 1 , but they performed significantly better than chance on Trial 2 and on Trials 3-5. Figure 1 shows the percentages of correct responses by Rat 1 (the rat with the clearest separation between Trial 1 and Trial 2 performances) and the median performances of the 3 rats separately for Trial 1 , Trial 2, and Trials 3-5 for each block of 20 five-trial oddity problems. As Figure 1 suggests, the differences seen as a function of Trial 1 , Trial 2 , and Trials 3-5 stabilized early in training and continued to be evident at the end of training.

The statements about significant differences above and below were based on an analysis of variance (ANOVA) that used as the scores to be analyzed the percentages correct on Trials 1,2 , and 3-5 for the 15 blocks of 20 problems. These data were analyzed separately for the 3 subjects; that is, subjects was treated as a between vari-

Table 3

Trials to Criterion by Each Rat, and Median Trials to Criterion on the Five Pretraining Problems in Experiment 2

\begin{tabular}{ccccc} 
& \multicolumn{3}{c}{ Rat } & \\
\cline { 2 - 5 } Problem & L1 & L2 & L3 & Median \\
\hline 1 & 100 & 60 & 80 & 80 \\
2 & 60 & 60 & 40 & 60 \\
3 & 20 & 40 & 40 & 40 \\
4 & 40 & 20 & 80 & 40 \\
5 & 20 & 20 & 20 & 20 \\
\hline
\end{tabular}



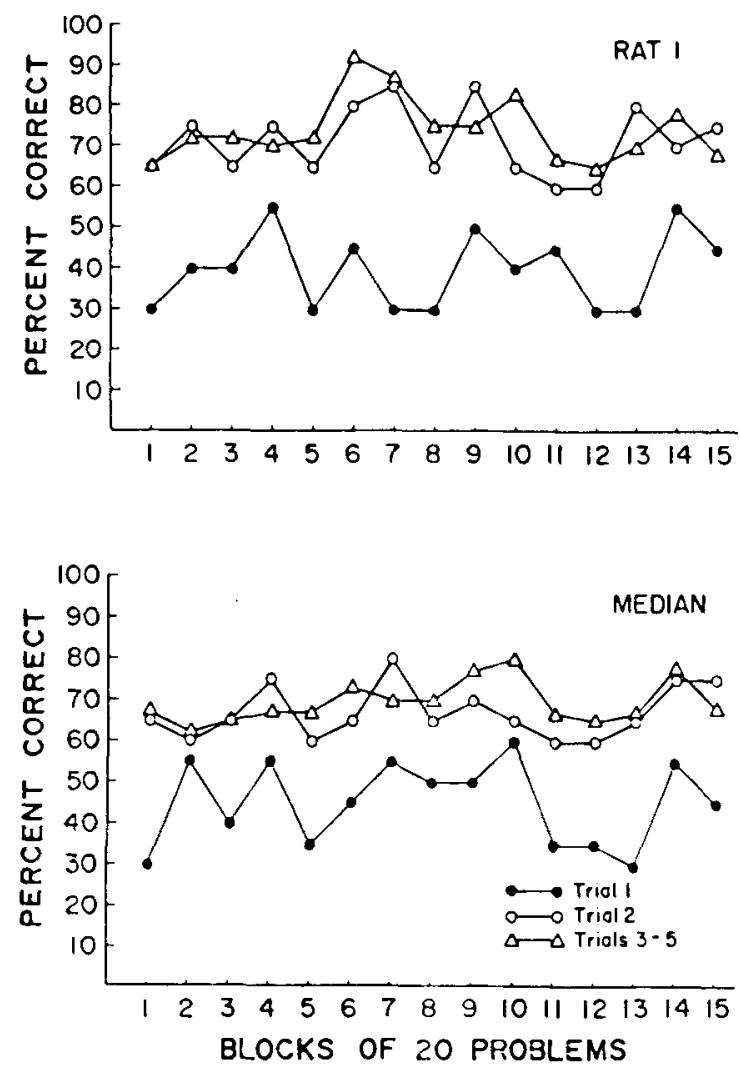

Figure 1. Percentages correct on Trial 1, Trial 2, and Trials 3-5 for Rat 1 (upper panel), and the median for Rats 1-3 as a function of blocks of $\mathbf{2 0}$ five-trial problems (lower panel).

able and trials (1, 2, and 3-5) as a within variable. The differences among subjects (Rat 1 was correct on $62 \%$ of all trials, Rat 2 on $58 \%$, and Rat 3 on $62 \%$ ) were not significant, but the differences among trials $[F(2,84)=$ $108.45, p<.001]$, and the subjects $\times$ trials interaction $[F(4,84)=8.085, p<.001]$, were significant.

A posteriori multiple-comparison tests were done with the Tukey HSD test (both the ANOVA and the multiplecomparisons tests were done with the computer programs provided by Pittenger \& Hodge, 1986). With an alpha of .01 , the overall difference between Trial $1(45 \%$ correct) and Trial 2 (67\% correct) and between Trial 1 and Trials 3-5 (70\% correct) was significant, but the difference between Trial 2 and Trials 3-5 was not significant. The mean percentage correct for Rat 1 on Trial $1(40 \%)$ differed significantly from that on Trial $2(71 \%)$ and on Trials 3-5 (74\%), but the difference between Trial 2 and Trials 3-5 was not significant. Similarly, the mean percentage correct for Rat 3 on Trial $1(48 \%)$ differed significantly from those on Trial $2(71 \%)$ and Trials 3-5 (69\%), but not between Trial 2 and Trials 3-5. For Rat 2 , there was a significant difference between the percentage correct on Trial 1 (48\%) and on Trials 3-5 (67\%), but the differences between Trial 1 and Trial $2(57 \%)$ and between Trial 2 and Trials 3-5 were not significant.

Whether 300 five-trial problems are sufficient to establish a conceptual response to oddity is not known, but it is relevant to note that the rats reached asymptote in their performances on Trials 2-5 very early in training (see Figure 1). If the significant intraproblem learning that was seen had been based on the use of the oddity concept, then the use of the oddity concept should have been evident on the first trials of new problems within the 300 problems provided.

The percentages correct on Trials 2-5 show that learning sets were well established for the 3 rats, which suggests that a high degree of training transfer was evident. The animals had learned well to use the information acquired on the first trial of a new problem to gain the reinforcers on subsequent trials of the same problem.

\section{GENERAL DISCUSSION}

The strong evidence seen in Experiment 2 that the rats had acquired learning sets shows that the stimuli, the response requirements, the reinforcers used, and the general characteristics of the testing situation were suitable for testing the rats. Although the evidence shows that they were able to acquire object-quality learning sets, there is no evidence to show that they were able to respond to the odd stimulus on a conceptual basis. Had they been able to do that, they should have been able to perform better than chance on the first trials with new oddity problems.

Successful learning-set performance is said to involve a kind of rule learning, a rule that has been characterized as "win stay-lose shift" (e.g., Levine, 1965). The application of this rule means that the subject stays with the first stimulus chosen if its response to that stimulus is reinforced (wins); the subject shifts to another stimulus if its response is not reinforced. To use the rule, the subject need only associate the specific physical properties of the stimuli with reinforcement. In the present study, had the rats associated the relational property of oddity with reinforcement, they should have been able to use that association to respond correctly whenever an oddity problem was presented, including first trials with new problems.

The distinction between responding to specific physical properties as opposed to conceptual properties must be considered in regard to any report of the use of a concept by an animal. If the experimental procedures and data analyses do not permit this distinction to be made unequivocably in favor of conceptual responding, then the report of conceptual responding is inconclusive.

A reviewer of the manuscript for this article raised the interesting question of whether the "novelty" of the stimuli on the first trials of new problems might have been aversive and caused the rats to fail in their first-trial performances. Two objections can be noted to this possibility. First, in both experiments, the rats did respond typically on the first trials, and all the stimuli were new. Although we did not record response times, it did not appear that the rats were any more hesitant on first trials than on subsequent trials. Second, in Experiment 2-the one more critical to the first-trial argument-only 16 odors 
were used. The rats had been exposed to all the odors early in training. What was new about the first trials was the combination of odors. Furthermore, except for wintergreen, the odors were food smells, which, it is reasonable to believe, the rats would be more likely to investigate than to avoid. Finally, "new" may be a more appropriate way to conceptualize the stimuli than "novel," as all stimuli were comparable in kind and, as noted, only 16 discriminable stimuli were used in Experiment 2.

None of the studies investigating the pigeon's use of the oddity concept have reported first-trial versus subsequent trials performances with new oddity problems (for references, see Carter \& Werner, 1978; Lombardi et al., 1984; Pisacreta et al., 1985). As the present study demonstrates, without such an analysis it is unwise to conclude that an animal used the oddity concept merely on the basis of facilitated transfer data. Such facilitated transfer might involve the learning-set process together with responses to specific physical properties of the stimuli rather than to the conceptual manifestations of those stimuli.

Phylogenetically, it is of considerable interest to determine which species can perform conceptual oddity, because oddity is a good example of a relative class concept. Although several species of nonprimate animals have successfully performed tasks that embody absolute class concepts, the evidence is weak that nonprimate animals can respond successfully on tasks that embody relative class concepts. The distinction between absolute and relative class concepts is based on the operational criterion of whether it is necessary to compare stimulus choices (Thomas \& Crosby, 1977). With absolute class concepts, such as "tree," it is not necessary to compare stimulus choices to affirm one that is an exemplar of class "tree," but with relative class concepts, such as oddity, it is necessary to compare stimulus choices to affirm the one that is the exemplar of oddity.

Only the study by Lombardi et al. (1984) using pigeons, and perhaps the study by Langworthy and Jennings (1972) using rats, have shown the conceptual use of oddity by a nonprimate animal. Lombardi et al.'s evidence involved better-than-chance performances on a test set of oddity problems, responses to which were never reinforced. Because responses to the test problems were never reinforced, the better-than-chance responding was presumably based on transfer of the oddity concept acquired in conjunction with a training set for which responses to the odd stimuli were reinforced. Whether the study by Langworthy and Jennings demonstrated conceptual responding to oddity by rats depends on whether nonconceptual odor cues were used to locate the reinforcers (see the introduction to Experiment 2). Even so, these two studies involve easier tests of the oddity concept than some that have been performed successfully by primates (see Thomas \& Frost, 1983).
Finally, the general importance of the oddity concept may be seen in a view that was expressed several times by Henry Nissen (e.g., 1958, p. 194):

All reasoning reduces to three processes: responsiveness to identity and to difference, and thirdly, the balance or relative weight given to each of these.... All class concepts require simultaneous responsiveness to identities among members of the class, and to differences between them and members of other classes. The balance between the two we may call sagacity; "judgment"' might be an even better term.

Perhaps the most intriguing aspect of Nissen's view is that concerned with the "relative balance" between responsiveness to identity and difference. Among tasks used with nonhuman animals, relative balance appears to have been addressed most clearly in "dimensionabstracted oddity" (DAO) tasks in which the nonodd stimuli are not identical, but do share more properties with each other than with the odd stimulus (e.g., Bernstein, 1961; Strong, Drash, \& Hedges, 1968; Thomas \& Frost, 1983), and in the more abstract versions of samenessdifference tasks (Premack, 1983; Smith, King, Witt, \& Rickel, 1975). Although primates perform successfully on such tasks, apparently no attempts have been made to use DAO or the more abstract sameness-difference tasks with nonprimate animals.

Despite his iteration of this viewpoint in several published sources, Nissen apparently never developed it further. Its validity is perhaps evident from the role that such processes have in standardized human intelligence tests (e.g., the Pictorial Similarities and Differences subtest of the Stanford-Binet; Terman \& Merrill, 1960) and in theories of the structure of intelligence (e.g., Jensen's, 1981, contemporary discussion of Spearman's $g$ and the measures that are highly correlated with it).

\section{REFERENCES}

Bernstein, I. S. (1961). The utilization of visual cues in dimensionabstracted oddity by primates. Joumal of Comparative \& Physiological Psychology, 54, 243-247.

Carter, D. E., \& Werner, T. J. (1978). Complex learning and information processing by pigeons: A critical analysis. Joumal of the $E x$ perimental Analysis of Behavior, 29, 565-601.

Fellows, B. J. (1967). Chance stimulus sequences for discrimination tasks. Psychological Bulletin, 67, 87-92.

FrenCH, G. M. (1965). Associative problems. In A. M. Schrier, H. F. Harlow, \& F. Stollnitz (Eds.), Behavior of nonhuman primates: I (pp. 167-209). New York: Academic Press.

HaRLow, H. F. (1949). The formation of learning sets. Psychological Review, 56, 51-65.

JENSEN, A. R. (1981). Straight talk about mental tests. New York: Free Press.

Koronakos, C., \& ARNold, W. J. (1957). The formation of learning sets in rats. Journal of Comparative \& Physiological Psychology, 50, 11-14.

LANGWorthy, R. A., Jennings, J. W. (1972). Odd ball, abstract, olfactory learning in laboratory rats. Psychological Record, 22, $487-490$.

Levine, M. (1965). Hypothesis behavior. In A. M. Schrier, H. F. 
Harlow, \& F. Stollnitz (Eds.), Behavior of nonhuman primates. I (pp. 97-127). New York: Academic Press.

Lombardi, C. M., Fachinelli, C. C., \& Deluus, J. D. (1984). Oddity of visual patterns conceptualized by pigeons. Animal Learning \& Behavior, 12, 2-6.

MEYER, D. R., \& HARLOW, H. F. (1949). The development of transfer of response to patterning by monkeys. Journal of Comparative \& Physiological Psychology, 42, 454-462.

Meyer, D. R., Treichler, F. R., \& Meyer, P. M. (1965). Discretetrial training techniques and stimulus variables. In A. M. Schrier, H. F. Harlow, \& F. Stollnitz (Eds.), Behavior of nonhuman primates: I (pp. 1-49). New York: Academic Press.

MEYER, M. E. (1976). A statistical analysis of behavior. Belmont, CA: Wadsworth.

Nissen, H. W. (1958). Axes of behavioral comparison. In A. Roe \& G. G. Simpson (Eds.), Behavior and evolution (pp. 183-205). New Haven, CT: Yale University Press.

PASTORE, N. (1954). Discrimination learning in the canary. Journal of Comparative \& Physiological Psychology, 47, 389-390.

Pisacreta, R., Lefave, P., Lesneski, T., \& Potter, C. (1985). Transfer of oddity learning in the pigeon. Animal Learning \& Behavior, 13, 403-414.

PtTtenger, D. J., \& Hodge, M.H. (1986). Statistical programs for Commodore computers. Behavior Research Methods, Instruments, \& Computers, 18, 50-52.

Premack, D. (1983). The codes of man and beasts. Behavioral \& Brain Sciences, 6, 125-167.

Robinson, E. W. (1933). A preliminary experiment on abstraction in a monkey. Journal of Comparative Psychology, 16, 231-236.

Smith, H. J., KING, J. E., WITT, E. D., \& RickEL, J. E. (1975).
Sameness-difference matching from sample by chimpanzees. Bulletin of the Psychonomic Society, 6, 469-471.

Strong, P. N., JR., Drash, P., \& Hedges, M. (1968). Solution of dimension abstracted oddity as a function of species, experience and intelligence. Psychonomic Science, 11, 337-338.

Strong, P. N., JR. \& HedGes, M. (1966). Comparative studies in simple oddity learning: I. Cats, raccoons, monkeys, and chimpanzees. Psychonomic Science, 5, 13-14.

Terman, L. M., \& Merrill, M. A. (1960). Stanford-Binet Intelligence Scale. Boston: Houghton Mifflin.

Thomas, R. K. (1980). Evolution of intelligence: An approach to its assessment. Brain, Behavior \& Evolution, 17, 452-474

Thomas, R. K., \& Boyd, M. G. (1973). A comparison of Cebus albifrons and Saimiri sciureus on oddity performance. Animal Learning \& Behavior, 1, 151-153.

ThomAs, R. K. \& Crosby, T. N. (1977). Absolute and relative class conceptual behavior in squirrel monkeys (Saimiri sciureus). Animal Learning \& Behavior, 5, 265-271.

ThомAS, R. K., \& Frost, T. (1983). Oddity and dimension-abstracted oddity (DAO) in squirrel monkeys. American Journal of Psychology, 96, 51-64.

WARREN, J. M. (1960). Oddity learning in the cat. Journal of Comparative \& Physiological Psychology, 53, 433-434.

Wodinsky, J., \& BitTerman, M. E. (1953). The solution of oddity problems by the rat. American Journal of Psychology, 66, 137-140.

ZeNTALL, T. R., \& HoGAN, D. E. (1974). Abstract concept learning in the pigeon. Journal of Experimental Psychology, 102, 393-398.

(Manuscript received January 29, 1987; revision accepted for publication December $5,1987$. ) 